\title{
Improved Design of Two and Four-group Decodable STBCs with Larger Diversity Product for Eight Transmit Antennas
}

\author{
Wei Liu ${ }^{1,2}$, Mathini Sellathurai ${ }^{1}$, Pei Xiao ${ }^{1}$, Chaojing Tang ${ }^{2}$, Jibo Wei ${ }^{2}$
}

\begin{abstract}
Recently, full rate and full diversity two-group (2Gp) and four-group (4Gp) decodable space-time block codes (STBC) derived from quasi-orthogonal STBC (QSTBC) and designed under diversity product maximization criterion have been proposed. In this paper, we derive an upper bound of diversity product for those STBCs and discover that the diversity product of the current $2 \mathrm{Gp}-\mathrm{QSTBC}$ and 4Gp-QSTBC has the potential to approach the upper bound for 8 transmit antennas. To this end, we propose an improved design of $2 \mathrm{Gp}$ and 4Gp STBC with increased diversity product for 8 transmit atennas by allowing sufficient number of dimensions for constellation rotation. Especially, the diversity product achieves the upper bound for the proposed two-group decodable STBC.

Keywords- quasi-orthogonal space-time block code, diversity product, two-group decodable STBC, four-group decodable STBC, constellation rotation, degrees of freedom
\end{abstract}

\section{INTRODUCTION}

Orthogonal Space-Time Block Codes (OSTBC) [1] provide a promising transmission scheme in multi-antenna systems due to their full diversity and single-symbol maximum likelihood (ML) decoding. However, it is proven in [1] that their symbol rates are less than one when complex signal constellations and more than two transmit antennas are used. To increase the rate, the quasi-orthogonal STBCs (QSTBC) [2] were proposed by relaxing the orthogonality constraint. The full diversity can still be obtained by rotating the constellations of parts of the transmitted symbols [3]. In the case of eight transmit antennas, a full rate (rate one) and full diversity $2 \mathrm{Gp}-\mathrm{QSTBC}$ with constellation rotation was presented in [4]. The symbols are separated into two groups and joint decoding of four symbols is performed. To reduce the decoding complexity, a coordinate interleaved orthogonal design (CIOD) with double symbols joint decoding was introduced in [5]. Recently, a full rate and full diversity 4GpQSTBC, with reduced peak-to-average power ratio (PAPR) in contrast to CIOD, has been proposed in [6], where double symbols are jointly decoded for eight transmit antennas since the transmitted symbols can be decoupled into 4 groups.

We focus on the analysis the 2Gp-QSTBC [4] and 4GpSTBC [6] for eight transmit antennas in this paper. They can be considered as QSTBCs with constellation rotation (CR) and interleaving the real and imaginary parts of different symbols before space-time coding. Both of the processes can be performed by an unitary transformation. The encoding process of $2 \mathrm{Gp}-\mathrm{QSTBC}$ and $4 \mathrm{Gp}-\mathrm{STBC}$ can be expressed as

1) The authors are with the Institute of Electronics, Communications, and Information Technology (ECIT), Queen's University of Belfast, Belfast BT3 9DT, U.K. (e-mail: \{wei.liu, m.sellathurai, pei.xiao\} @ qub.ac.uk). 2) The authors are with the School of Electronic Science and Engineering, National University of Defense Technology, Changsha, 410073, China. (email: \{wliu_nudt,wjbhw\}@nudt.edu.cn).

$$
\begin{array}{r}
\mathbf{C}=f(\mathbf{x}) \\
\mathbf{x}=\mathbf{U}_{\mathbf{G p}} \mathbf{a}
\end{array}
$$

where $\mathbf{C}$ is an $8 \times 8$ space-time codeword, $f(\bullet)$ is encoder of QSTBC, $\mathbf{U}_{\mathbf{G p}}$ is a $16 \times 16$ unitary matrix for $\mathrm{CR}$ and interleaving, and $\quad \mathbf{a}=\left[\begin{array}{llllll}a_{1 I} & \ldots & a_{K I} & a_{1} Q & \ldots & a_{K Q}\end{array}\right]^{T} \quad$ and

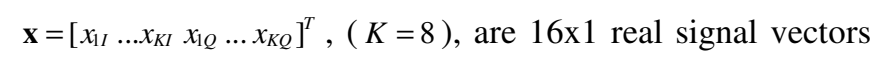
composed of the real and imaginary parts of $a_{k}$ and $x_{k}$, respectively. The superscript ()$^{T}$ denote matrix transpose. We define $a_{k}=a_{k I}+j a_{k Q}$ and $x_{k}=x_{k I}+j x_{k Q}, \quad(k=1,2, \ldots, 8)$, where $j=\sqrt{-1}$, and the subscript ( $)_{I}$ and ()$_{Q}$ denote the real and imaginary part of a complex scalar, respectively.

It was shown in [7] that QSTBC symbols transmitted from different antennas can be completely decoupled if they are pre-processed by a decoupling matrix. The decoupling process can be expressed as

$$
\mathbf{x}=\mathbf{V s}
$$

where $\mathbf{V}$ is a real constant unitary matrix, and $\mathbf{s}=\left[\begin{array}{lllll}s_{1 I} & \ldots & s_{K I} s_{1 Q} & \ldots & s_{K Q}\end{array}\right]^{T}, s_{k}=s_{k I}+j s_{k Q}, k=1,2, \ldots, 8$. We can now define a new encoder as $g(\bullet)$, which is a concatenation of $\mathbf{V}$ and $f(\bullet)$ as shown in Fig. 1. If there is no correlation between the real symbols $\left\{s_{1 I} \ldots s_{K I} s_{1 Q} \ldots s_{K Q}\right\}$, we can employ single real symbol ML decoding in the receiver [8]. However its transmit diversity order is only 2 .

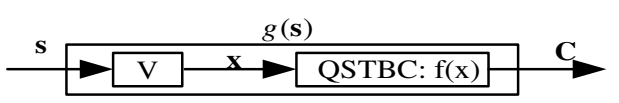

Fig. 1. Coding structure with decoupling characterization

We consider a class of STBCs with the unified framework as

$$
\begin{aligned}
\mathbf{C} & =g(\mathbf{s}) \\
\mathbf{s} & =\mathbf{U} \mathbf{a}
\end{aligned}
$$

where $\mathbf{U}$ is a real unitary matrix, such as $\mathbf{U}=\mathbf{V}^{\mathbf{T}}$ for QSTBCs, $\mathbf{U}=\mathbf{I}$ for the single-symbol decodable STBC proposed in [7], and $\mathbf{U}=\mathbf{V}^{T} \mathbf{U}_{\text {Gp }}$ for 2Gp-QSTBC [4] or 4Gp-QSTBC [6]. In this paper, we propose an improved design for two-group and four-group decodable STBC based on the unified framework with increased diversity product for eight transmit antennas. The contributions of this paper are:

- Derivation of an upper bound of diversity product for those STBCs designed under the framework in (4) and (5).

- Consideration of sufficient number of rotation dimensions to allow more freedom in search for larger diversity product. 
- Construction of a new four-group decodable STBC based on four-dimensional rotation with a larger diversity product but the same complexity as double symbols joint ML decoding.

- Construction of a two-group decodable STBC, which has the same complexity as four symbols joint ML decoding, but achieves the upper bound of diversity product.

Notation: The superscript ()$^{H}$ denotes transpose conjugate of a matrix (or vector). || stands for norm of a vector. The identity square matrix and zero matrix of proper size are denoted by $\mathbf{I}$ and $\mathbf{0}$, respectively. $\operatorname{Re}(\mathbf{X})$ and $\operatorname{Im}(\mathbf{X})$ denote the real and imaginary parts of $\mathbf{X}$, respectively. $\operatorname{Rank}(\mathbf{X})$ and Trace $(\mathbf{X})$ denote the rank and trace of $\mathbf{X} . \operatorname{vec}(\mathbf{X})$ denotes the vectorization operation of matrix $\mathbf{X}$, i.e., $\operatorname{vec}(\mathbf{X})=\left[\mathbf{X}_{1}^{T} \mathbf{X}_{2}^{T} \ldots \mathbf{X}_{n}^{T}\right]^{T}$, where $\mathbf{X}_{i}$ is the $i^{\text {th }}$ column of $\mathbf{X}$.

\section{AlGEBRAIC STRUCTURE OF ENCODER $g(\mathbf{s})$}

Let $N_{T}$ be the number of transmit antennas, $N_{R}$ the number of receive antennas, and $T$ the number of time slots for one codeword. The number of transmitted symbols in each codeword is $K$. Here, we focus on QSTBC with $T=N_{T}=K=8$. Without loss of generality, we consider the following codeword:

$$
f(\mathbf{x})=\left[\begin{array}{cccccccc}
x_{1} & x_{2} & x_{3} & x_{4} & x_{5} & x_{6} & x_{7} & x_{8} \\
x_{2}^{*} & -x_{1}^{*} & x_{4}^{*} & -x_{3}^{*} & x_{6}^{*} & -x_{5}^{*} & x_{8}^{*} & -x_{7}^{*} \\
x_{3} & -x_{4} & -x_{1} & x_{2} & x_{7} & -x_{8} & -x_{5} & x_{6} \\
x_{4}^{*} & x_{3}^{*} & -x_{2}^{*} & -x_{1}^{*} & x_{8}^{*} & x_{7}^{*} & -x_{6}^{*} & -x_{5}^{*} \\
x_{5} & -x_{6} & x_{7} & -x_{8} & -x_{1} & x_{2} & -x_{3} & x_{4} \\
x_{6}^{*} & x_{5}^{*} & x_{8}^{*} & x_{7}^{*} & -x_{2}^{*} & -x_{1}^{*} & -x_{4}^{*} & -x_{3}^{*} \\
x_{7} & x_{8} & -x_{5} & -x_{6} & -x_{3} & -x_{4} & x_{1} & x_{2} \\
x_{8}^{*} & -x_{7}^{*} & -x_{6}^{*} & x_{5}^{*} & -x_{4}^{*} & x_{3}^{*} & x_{2}^{*} & -x_{1}^{*}
\end{array}\right]
$$

According to the analysis in [7], we can obtain its decoupling matrix as

$$
\mathbf{V}=\left[\begin{array}{cc}
\operatorname{Re}\left(\mathbf{V}^{\prime}\right) & -\operatorname{Im}\left(\mathbf{V}^{\prime}\right) \\
\operatorname{Im}\left(\mathbf{V}^{\prime}\right) & \operatorname{Re}\left(\mathbf{V}^{\prime}\right)
\end{array}\right]
$$

where

$$
\mathbf{V}^{\prime}=\frac{1}{2}\left[\begin{array}{cccccccc}
1 & 0 & 1 & 0 & 1 & 0 & 1 & 0 \\
0 & 1 & 0 & 1 & 0 & 1 & 0 & 1 \\
-j & 0 & -j & 0 & j & 0 & j & 0 \\
0 & j & 0 & j & 0 & -j & 0 & -j \\
-j & 0 & j & 0 & -j & 0 & j & 0 \\
0 & j & 0 & -j & 0 & j & 0 & -j \\
-1 & 0 & 1 & 0 & 1 & 0 & -1 & 0 \\
0 & -1 & 0 & 1 & 0 & 1 & 0 & -1
\end{array}\right]
$$

Substituting (3) into (6), the dispersion form of the codeword $g(\mathbf{s})$ can be written as

$$
\mathbf{C}=g(\mathbf{s})=\sum_{k=1}^{K} s_{k I} \mathbf{A}_{k}+s_{k Q} \mathbf{A}_{k+K}
$$

where $\left\{\mathbf{A}_{l}\right\}_{l=1}^{2 K}$ is a set of $T \times N_{T}$ complex matrices called dispersion matrices of $g(\mathbf{s})$. According to our analysis, they satisfy the following conditions:

$$
\begin{array}{ll}
\operatorname{Trace}\left(\mathbf{A}_{m}^{H} \mathbf{A}_{l}\right)=0, & 1 \leq m \neq l \leq 2 K \\
\operatorname{Trace}\left(\mathbf{A}_{l}^{H} \mathbf{A}_{l}\right)=N_{T}, & 1 \leq l \leq 2 K
\end{array}
$$

The codeword in (8) can be reformed in a vector form as

$$
\operatorname{vec}(\mathbf{C})=\mathbf{G s}
$$

where $\mathbf{G}$ is a encoding matrix [9] defined as

$$
\mathbf{G}=\left[\operatorname{vec}\left(\mathbf{A}_{1}\right) \operatorname{vec}\left(\mathbf{A}_{2}\right) \ldots \operatorname{vec}\left(\mathbf{A}_{2 K}\right)\right]
$$

From (9), (10) and (12), we have

$$
\mathbf{G}^{H} \mathbf{G}=N_{T} \mathbf{I}
$$

\section{UPPER BOUND OF DIVERSITY PRODUCT}

In this section, we shall derive an upper bound of diversity product for STBCs designed under the framework in (4) and (5). Assuming the difference codeword as $\Delta \mathbf{C}=\mathbf{C}-\mathbf{C}^{\prime}$, if $\Delta \mathbf{C}^{H} \Delta \mathbf{C}$ is of full rank for any pair of distinct codewords $\mathbf{C}$ and $\mathbf{C}^{\prime}$, the diversity product [3] is given by

$$
\zeta=\frac{1}{2 \sqrt{N_{T}}} \min _{C \neq C^{\prime}}\left|\operatorname{det}\left(\Delta \mathbf{C}^{H} \Delta \mathbf{C}\right)\right|^{1 /(2 T)}
$$

According to the theory of inequality, we know that

$$
\left|\operatorname{det}\left(\Delta \mathbf{C}^{H} \Delta \mathbf{C}\right)\right| \leq\left(\frac{\operatorname{Trace}\left(\Delta \mathbf{C}^{H} \Delta \mathbf{C}\right)}{N_{T}}\right)^{N_{T}}
$$

From (11), (13) and (5) we can derive

$$
\operatorname{Trace}\left(\Delta \mathbf{C}^{H} \Delta \mathbf{C}\right)=(\operatorname{vec}(\Delta \mathbf{C}))^{H}(\operatorname{vec}(\Delta \mathbf{C}))=N_{T}|\Delta \mathbf{a}|^{2}
$$

where $\quad \Delta \mathbf{a}=\mathbf{a}-\mathbf{a}^{\prime}, \quad \mathbf{a} \neq \mathbf{a}^{\prime} \quad$ and $\quad \mathbf{a}=\left[a_{1 I} \ldots a_{K I} a_{1 Q} \ldots a_{K Q}\right]^{T}$,

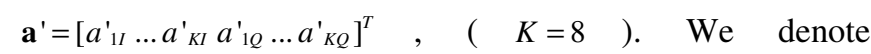
$a_{k}=a_{k l}+j a_{k Q}$ and $a_{k}^{\prime}=a_{k l}^{\prime}+j a_{k Q}^{\prime}, \quad(k=1,2, \ldots, K)$ are the signals from known constellations, e.g. quadratureamplitude-modulated (QAM). Obviously, the minimum Euclidean distance between two distinct points in the signal constellation is

$$
d_{\min }=\min _{a \neq a^{\prime}}|\Delta \mathbf{a}|
$$

Combining (14) (17) and $T=N_{T}$, we obtain the upper bound of diversity product as

$$
\zeta_{u b}=\frac{d_{\min }}{2 \sqrt{N_{T}}}
$$

The upper bound of diversity product is uniquely determined by $d_{\min }$.

\section{PROPOSED CODES}

Both the current 4Gp-QSTBC and 2Gp-QSTBC obtain their diversity product by optimal constellation rotation with 2 and 4 degrees of freedom, respectively. However, their diversity products deviates from the bound given in (18) for $N_{T}=8$ by a large margin because their rotation dimensions are not sufficient. In this section, we design two improved four-group and two-group decodable STBCs to approach the bound by a rotation matrix with 6 dimensions.

\section{A. Proposed four-group decodable STBC}

We divide the symbols $\left\{s_{1 I}, \ldots, s_{K I}, s_{1 Q}, \ldots, s_{K Q}\right\}$ into four groups as $\widehat{\mathbf{s}}_{1}=\left[\begin{array}{llll}s_{1 I} & s_{3 I} & s_{5 I} & s_{7 I}\end{array}\right]^{T}, \quad \widehat{\mathbf{s}}_{2}=\left[\begin{array}{llll}s_{1 Q} & s_{3 Q} & s_{5 Q} & s_{7 Q}\end{array}\right]^{T}$, $\widehat{\mathbf{s}}_{3}=\left[\begin{array}{llll}s_{2 I} & s_{4 I} & s_{6 I} & s_{8 I}\end{array}\right]^{T}, \widehat{\mathbf{s}}_{4}=\left[\begin{array}{llll}s_{2 Q} & s_{4 Q} & s_{6 Q} & s_{8 Q}\end{array}\right]^{T}$, and let

$$
\widehat{\mathbf{s}}_{k}=\widehat{\mathbf{U}}_{k} \widehat{\mathbf{a}}_{k}, k=1,2,3,4
$$

where $\widehat{\mathbf{a}}_{1}=\left[\begin{array}{llll}a_{1 I} & a_{2 I} & a_{1 Q} & a_{2 Q}\end{array}\right]^{T}, \quad, \quad \hat{\mathbf{a}}_{2}=\left[\begin{array}{llll}a_{3 I} & a_{4 I} & a_{3 Q} & a_{4 Q}\end{array}\right]^{T}$, $\widehat{\mathbf{a}}_{3}=\left[\begin{array}{llll}a_{5 I} & a_{61} & a_{5 Q} & a_{6 Q}\end{array}\right]^{T}, \widehat{\mathbf{a}}_{4}=\left[\begin{array}{llll}a_{71} & a_{8 I} & a_{7 Q} & a_{8 Q}\end{array}\right]^{T}$, and $\widehat{\mathbf{U}}_{k}$ is a real unitary matrix.

Combining the expressions in (3), (6) and (7), we obtain 


$$
\begin{aligned}
& \left|\operatorname{det}\left(\Delta \mathbf{C}^{H} \Delta \mathbf{C}\right)\right|= \\
& 4^{8} \times\left(\left(\Delta s_{1 I}{ }^{2}+\Delta s_{1 Q}{ }^{2}+\Delta s_{2 I}{ }^{2}+\Delta s_{2 Q}{ }^{2}\right) \bullet\right. \\
& \left(\Delta s_{3 I}{ }^{2}+\Delta s_{3 Q}{ }^{2}+\Delta s_{4 I}{ }^{2}+\Delta s_{4 Q}{ }^{2}\right) \bullet \\
& \left(\Delta s_{5 I}{ }^{2}+\Delta s_{5 Q}{ }^{2}+\Delta s_{6 I}{ }^{2}+\Delta s_{6 Q}{ }^{2}\right) \bullet \\
& \left.\left(\Delta s_{7 I}{ }^{2}+\Delta s_{7 Q}{ }^{2}+\Delta s_{8 I}{ }^{2}+\Delta s_{8 Q}{ }^{2}\right)\right)^{2}
\end{aligned}
$$

where $\Delta s_{k I}=s_{k I}-s_{k I}^{\prime}, \quad \Delta s_{k Q}=s_{k Q}-s_{k Q}^{\prime}, \quad s_{k}=s_{k I}+j s_{k Q}$, $s^{\prime}{ }_{k}=s^{\prime}{ }_{k I}+j s^{\prime}{ }_{k Q}, k=1,2, \ldots, 8$.

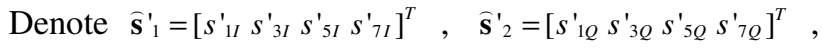

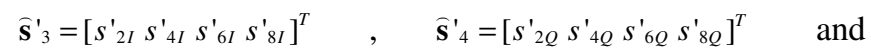
$\Delta \widehat{\mathbf{s}}_{k}=\widehat{\mathbf{s}}_{k}-\widehat{\mathbf{s}}^{\prime}{ }_{k}, \quad k=1,2,3,4$. To obtain the minimum of determinant expression in (20), without loss of generality, we can assume $\Delta \widehat{\mathbf{s}}_{2}=\Delta \widehat{\mathbf{s}}_{3}=\Delta \widehat{\mathbf{s}}_{4}=0$. Then (20) can be rewritten as

$$
\left|\operatorname{det}\left(\Delta \mathbf{C}^{H} \Delta \mathbf{C}\right)\right|=4^{8} \times\left(\Delta{s_{1 I}}^{2} \Delta s_{3 I}{ }^{2} \Delta s_{5 I}{ }^{2} \Delta s_{7 I}{ }^{2}\right)^{2}
$$

Equation (21) can be maximized for any $\Delta \widehat{\mathbf{S}}_{1} \neq 0$ with sufficient dimensions of constellation rotation. We consider $\widehat{\mathbf{U}}_{1}$ as a rotation matrix with $\left(\begin{array}{l}2 \\ 4\end{array}\right)=6$ degrees of freedom. A possible choice of $\hat{\mathbf{U}}_{1}$ is [10]

$$
\begin{gathered}
\widehat{\mathbf{U}}_{1}=\left[\begin{array}{cccc}
1 & 0 & 0 & 0 \\
0 & 1 & 0 & 0 \\
0 & 0 & c_{\theta 6} & -s_{\theta 6} \\
0 & 0 & s_{\theta 6} & c_{\theta 6}
\end{array}\right] \cdot\left[\begin{array}{cccc}
1 & 0 & 0 & 0 \\
0 & c_{\theta 5} & 0 & -s_{\theta 5} \\
0 & 0 & 1 & 0 \\
0 & s_{\theta 5} & 0 & c_{\theta 5}
\end{array}\right] \cdot\left[\begin{array}{cccc}
1 & 0 & 0 & 0 \\
0 & c_{\theta 4} & -s_{\theta 4} & 0 \\
0 & s_{\theta 4} & c_{\theta 4} & 0 \\
0 & 0 & 0 & 1
\end{array}\right] \\
\bullet\left[\begin{array}{cccc}
c_{\theta 3} & 0 & 0 & -s_{\theta 3} \\
0 & 1 & 0 & 0 \\
0 & 0 & 1 & 0 \\
s_{\theta 3} & 0 & 0 & c_{\theta 3}
\end{array}\right] \cdot\left[\begin{array}{cccc}
c_{\theta 2} & 0 & -s_{\theta 2} & 0 \\
0 & 1 & 0 & 0 \\
s_{\theta 2} & 0 & c_{\theta 2} & 0 \\
0 & 0 & 0 & 1
\end{array}\right] \bullet\left[\begin{array}{cccc}
c_{\theta 1} & -s_{\theta 1} & 0 & 0 \\
s_{\theta 1} & c_{\theta 1} & 0 & 0 \\
0 & 0 & 0 & 0 \\
0 & 0 & 0 & 0
\end{array}\right]
\end{gathered}
$$

where $c_{\theta i}=\cos \left(\theta_{i}\right)$ and $s_{\theta i}=\sin \left(\theta_{i}\right)$. Then we can obtain the optimal rotation angles $\theta_{1} \sim \theta_{6}$ that maximize $\min _{\bar{s}_{1} \neq \bar{s}_{1}}\left|\operatorname{det}\left(\Delta \mathbf{C}^{H} \Delta \mathbf{C}\right)\right|$ in (21) through exhaustive computer search. In the case of $\left\{a_{k}\right\}_{k=1}^{K}$ from conventional QAM constellation, we have

$\left[\begin{array}{llllll}\theta_{1} & \theta_{2} & \theta_{3} & \theta_{4} & \theta_{5} & \theta_{6}\end{array}\right]_{\text {opt }}=$

$\left[\begin{array}{lll}36.4145^{\circ} 20.1470^{\circ} 25.0290^{\circ} & -25.0290^{\circ} 20.1470^{\circ} & -25.5130^{\circ}\end{array}\right]$

Substituting (23) into (22) yields the optimal rotation matrix. Similarly, other $\hat{\mathbf{U}}_{k}, k=2,3,4$, can be selected as

$$
\hat{\mathbf{U}}_{1}=\hat{\mathbf{U}}_{2}=\hat{\mathbf{U}}_{3}=\hat{\mathbf{U}}_{4}
$$

Obviously, the four real symbols in each vector $\widehat{\mathbf{s}}_{k}$ are correlated due to the unitary transformation with the matrix $\hat{\mathbf{U}}_{k}$, but there is no correlation among the vectors $\widehat{\mathbf{s}}_{1} \sim \widehat{\mathbf{s}}_{4}$, each $\widehat{\mathbf{s}}_{k}$ can thus be decoded independently.

\section{B. Proposed two-group decodable STBC}

We divide the symbols $\left\{s_{1 I}, \ldots, s_{K I}, s_{1 Q}, \ldots, s_{K Q}\right\}$ into two groups as $\quad \hat{\mathbf{s}}_{1}=\left[\begin{array}{llllll}s_{1 I} & s_{3 I} s_{5 I} s_{7 I} s_{1 Q} s_{3 Q} s_{5 Q} s_{7 Q}\end{array}\right]^{T}$,

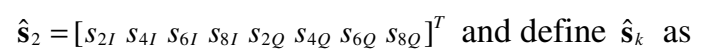

$$
\hat{\mathbf{s}}_{k}=\hat{\mathbf{U}}_{k} \hat{\mathbf{a}}_{k}, \quad k=1,2
$$

where $\hat{\mathbf{a}}_{1}=\left[\begin{array}{ll}\hat{\mathbf{a}}_{1}^{T} & \widehat{\mathbf{a}}_{2}^{T}\end{array}\right]^{T}, \hat{\mathbf{a}}_{2}=\left[\begin{array}{ll}\hat{\mathbf{a}}_{3}^{T} & \widehat{\mathbf{a}}_{4}^{T}\end{array}\right]^{T}$.

Define $\quad \hat{\mathbf{s}}_{1}{ }_{1}=\left[s_{{ }_{1 I}} s^{\prime}{ }_{3 I} s_{{ }_{5 I}}^{\prime} s_{7 I}^{{ }_{7 I}} s_{1 Q}^{{ }_{1 Q}} s_{3 Q}{ }_{3 Q} s_{5 Q} s_{7 Q}^{{ }_{7 Q}}\right]^{T}$,

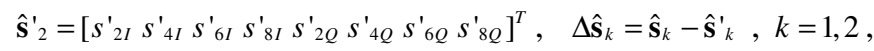
and assume that $\Delta \hat{\mathbf{s}}_{1} \neq 0$ and $\Delta \hat{\mathbf{s}}_{2}=0$. The determinant in (20) can now be rewritten as

$$
\begin{aligned}
\left|\operatorname{det}\left(\Delta \mathbf{C}^{H} \Delta \mathbf{C}\right)\right|= & 4^{8} \times\left[\left(\Delta s_{1 I}{ }^{2}+\Delta s_{1 Q}{ }^{2}\right) \square\left(\Delta s_{3 I}{ }^{2}+\Delta s_{3 Q}{ }^{2}\right) \square\right. \\
& \left.\left(\Delta s_{5 I}{ }^{2}+\Delta s_{5 Q}{ }^{2}\right) \square\left(\Delta s_{7 I}{ }^{2}+\Delta s_{7 Q}{ }^{2}\right)\right]^{2}
\end{aligned}
$$

If we consider $\hat{\mathbf{U}}_{1}$ as a rotation matrix with $\left(\begin{array}{l}2 \\ 8\end{array}\right)=28$ degrees of freedom, it becomes intractable to find out the 28 optimal angles by computer search due to the large computation. To tackle this problem, we limit the constellational rotation to 6 dimensions. The structure of $\hat{\mathbf{U}}_{1}$ is depicted in Fig. 2

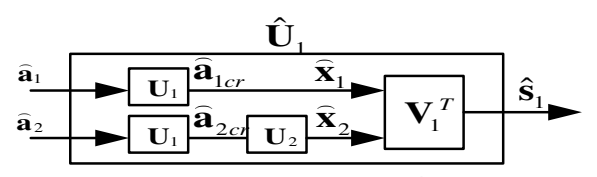

Fig. 2. Structure of $\hat{\mathbf{U}}_{1}$

Rotating the symbols $\left\{a_{2}, a_{4}\right\}$ with angle $\alpha$, while keeping the symbols $\left\{a_{1}, a_{3}\right\}$ un-rotated, we obtain

$$
\widehat{\mathbf{a}}_{k c r}=\mathbf{U}_{1} \widehat{\mathbf{a}}_{k}, \quad k=1,2
$$

where $\widehat{\mathbf{a}}_{k}$ is defined in (19), $\alpha=\pi / 4$ is a optimal rotation angle for conventional QAM proposed in [3], and

$$
\mathbf{U}_{1}=\left[\begin{array}{cccc}
1 & 0 & 0 & 0 \\
0 & \cos (\alpha) & 0 & -\sin (\alpha) \\
0 & 0 & 1 & 0 \\
0 & \sin (\alpha) & 0 & \cos (\alpha)
\end{array}\right]
$$

Similarly, we rotate $\widehat{\mathbf{a}}_{2 c r}$, and keep $\widehat{\mathbf{a}}_{1 c r}$ un-rotated, i.e.,

$$
\widehat{\mathbf{x}}_{1}=\widehat{\mathbf{a}}_{1 c r}, \quad \widehat{\mathbf{x}}_{2}=\mathbf{U}_{2} \hat{\mathbf{a}}_{2 c r}
$$

where $\mathbf{U}_{2}$ is a rotation matrix which is similar to (22) with $\theta_{1} \sim \theta_{6}$ replaced by $\varphi_{1} \sim \varphi_{6}$.

We define $\widehat{\mathbf{x}}_{1}$ and $\widehat{\mathbf{x}}_{2}$ as

$$
\widehat{\mathbf{x}}_{1}=\left[\begin{array}{llll}
x_{1 I} & x_{3 I} & x_{1 Q} & x_{3 Q}
\end{array}\right]^{T}, \widehat{\mathbf{x}}_{2}=\left[\begin{array}{llll}
x_{5 I} & x_{7 I} & x_{5 Q} & x_{7 Q}
\end{array}\right]^{T}
$$

which can be substituted into codeword (6) to derive the QSTBC code. Alternatively, placing it under the unified framework, the $\hat{\mathbf{s}}_{1}$ is given as

$$
\hat{\mathbf{s}}_{1}=\mathbf{V}_{1}^{T} \hat{\mathbf{x}}_{1}
$$

where $\hat{\mathbf{x}}_{1}=\left[\begin{array}{ll}\widehat{\mathbf{x}}_{1}^{T} & \widehat{\mathbf{x}}_{2}^{T}\end{array}\right]^{T}$, from the equations in (3), (7) and (31), we can derive

$$
\mathbf{V}_{1}=\frac{1}{2}\left[\begin{array}{cccccccc}
1 & 1 & 1 & 1 & 0 & 0 & 0 & 0 \\
0 & 0 & 0 & 0 & 1 & 1 & -1 & -1 \\
0 & 0 & 0 & 0 & 1 & 1 & 1 & 1 \\
-1 & -1 & 1 & 1 & 0 & 0 & 0 & 0 \\
0 & 0 & 0 & 0 & 1 & -1 & 1 & -1 \\
-1 & 1 & 1 & -1 & 0 & 0 & 0 & 0 \\
-1 & 1 & -1 & 1 & 0 & 0 & 0 & 0 \\
0 & 0 & 0 & 0 & -1 & 1 & 1 & -1
\end{array}\right]
$$

After knowing $\mathbf{U}_{1}$ and $\mathbf{V}_{1}$, we obtain the optimal angles to maximize $\min _{\hat{s}_{1} \neq \hat{s}_{1}}\left|\operatorname{det}\left(\Delta \mathbf{C}^{H} \Delta \mathbf{C}\right)\right|$ in (26) for conventional QAM constellation through computer search as follows $\left[\begin{array}{llllll}\varphi_{1} & \varphi_{2} & \varphi_{3} & \varphi_{4} & \varphi_{5} & \varphi_{6}\end{array}\right]_{\text {opt }}$

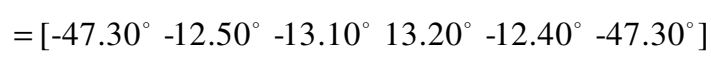

$\hat{\mathbf{U}}_{2}$ can be simply chosen as

$$
\hat{\mathbf{U}}_{2}=\hat{\mathbf{U}}_{1}
$$

Similar to our discussion in Section IV-A, $\hat{\mathbf{s}}_{1}$ and $\hat{\mathbf{s}}_{2}$ are un-correlated, therefore can be decoded independently. 


\section{NUMERICAL RESUlTS}

We consider data transmission over a quasi-static Rayleigh flat fading channel. The channel gains are assumed to be known at the receiver but not at the transmitter. The simulation results are presented in Fig. 3 to compare the bit error rate (BER) of proposed codes with the current $4 \mathrm{Gp}$ QSTBC [6], 2Gp-STBC [4] and CIOD [5] with 2 bits pcu (4QAM) for $8 \mathrm{Tx}$ and $1 \mathrm{Rx}$ antennas.

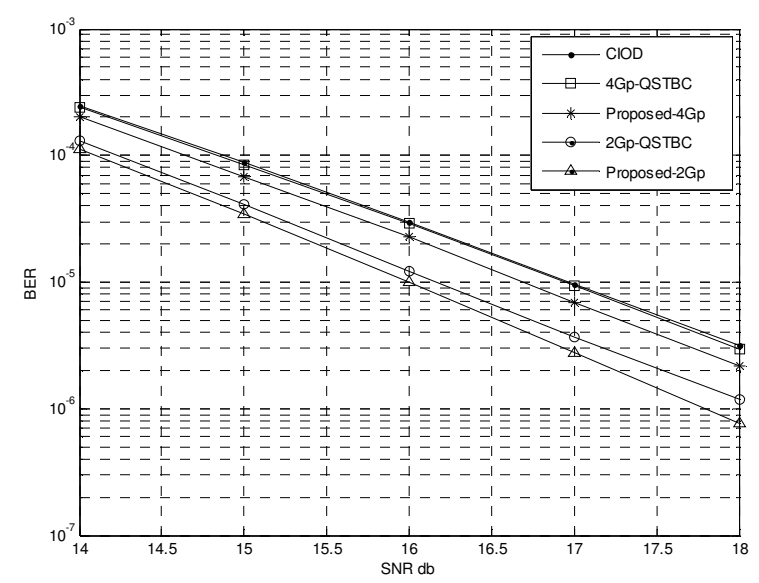

Fig. 3. Performance of proposed codes compared with current CIOD, 4Gp-QSTBC and 2Gp-QSTBC, 2 bits pcu.

It is shown that the proposed four-group decodable STBC outperforms the CIOD and $4 \mathrm{Gp}-\mathrm{QSTBC}$ by $0.25 \mathrm{~dB}$ with the same number of symbols for joint decoding. In the case of the two-group decodable STBCs, the proposed code also obtains about $0.25 \mathrm{~dB}$ gain compared to $2 \mathrm{Gp}$-QSTBC. Overall, the two-group STBCs have better performance than four-group decodable.

Table 1: Diversity product of STBCs for $\mathrm{Nt}=\mathrm{T}=8$

\begin{tabular}{|c|c|c|}
\hline & $\begin{array}{c}\text { Number of complex } \\
\text { symbols for joint decoding }\end{array}$ & $\begin{array}{c}\text { Diversity } \\
\text { product }\end{array}$ \\
\hline Upper Bound & & 0.2500 \\
\hline 2Gp-QSTBC [4] & 4 & 0.2187 \\
\hline Proposed 2Gp STBC & 4 & 0.2500 \\
\hline 4Gp-QSTBC [6] & 2 & 0.1727 \\
\hline CIOD[5],[11] & 2 & 0.1747 \\
\hline Proposed 4Gp STBC & 2 & 0.2195 \\
\hline
\end{tabular}

Table 1 shows the upper bound and some diversity products of the STBCs discussed previously with 4QAM signal constellation. It can be seen that the proposed schemes for two-group and four-group decodable STBC obtain higher diversity product than the existing ones. Especially, the proposed two-group decodable STBC is able to achieve the upper bound of diversity product.

It is stated in [11] that maximizing diversity product does not necessarily minimize the pairwise-error-probability (PEP)-bound for $8 \times 8$ STBCs. Considering our design by PEP-bound minimization criterion, we reach the following conclusions

- From Table 1, the proposed four-group decodable STBC has a little higher diversity product than $2 \mathrm{Gp}-\mathrm{QSTBC}$ but no better performance. The reason is that $2 \mathrm{Gp}$ QSTBC has a lower PEP-bound.

- Designing the proposed four-group decodable STBC under PEP-bound minimization criterion leads to 0.05 $\mathrm{dB}$ additional gain.

- When diversity product achieves its upper bound, all eigenvalues of $\Delta \mathbf{C}^{H} \Delta \mathbf{C}$ should be equal, so it is easy to approve that the PEP-bound must be minimum in this case.

\section{CONCLUSIONS}

We proposed an unified framework for the STBCs based on QSTBC and derived an upper bound of diversity product for this particular class of STBCs in this paper. An improved design of four-group and two-group decodable STBC have been proposed with better performance and the same decoding complexity in comparison to current 4Gp-QSTBC and $2 \mathrm{Gp}-\mathrm{QSTBC}$. We also concluded that it is necessary to exploit sufficient degrees of freedom for constellation rotation to facilitate the search of larger diversity product. The degrees of freedom are determined by the number of symbols in joint decoding.

\section{REFERENCES}

[1] V. Tarokh, H. Jafarkhani, and A. R. Calderbank, "Space-time block codes from orthogonal designs," IEEE Trans. Inform. Theory, vol. 45, pp. 1456-1466, July 1999.

[2] H. Jafarkhani, "A quasi-orthogonal space-time block code," IEEE Trans. Commun., vol. 49, pp. 1-4, January 2001.

[3] W. Su and X. G. Xia, "Signal constellations for quasiorthogonal spacetime block codes with full diversity," IEEE Trans. Inform. Theory, vol. 50, pp. 2331-2347, October 2004.

[4] N. Sharma and C. B. Papadias, "Full rate full diversity linear quasiorthogonal space-time codes for any transmit antennas," EURASIP Journal on Applied Signal Proc., pp. 1246-1256, August 2004.

[5] Z. A. Khan, M. H. Lee and B. S. Rajan, "A rate one full-diversity quasi-orthogonal design for eight Tx antennas," Internal Report, TRPME-2002-15, available at http://pal.ece.iisc.ernet.in/PAM/tech rep02.html.

[6] D. N. Dao, C. Yuen, C. Tellambura, Y. L. Guan and T. T. Tjhung, "Four-Group Decodable Space-Time Block Codes", IEEE Trans. Signal Processing, Vol. 56, pp. 424-430, January 2008.

[7] A. Sezgin and T. J. Oechtering, "Complete characterization of the equivalent MIMO Channel for quasi-orthogonal space-time codes," IEEE Trans. Inform. Theory, vol. 54, pp. 3315-3327, July 2008.

[8] W. Liu, M. Sellathurai, P. Xiao and J. Wei, "A New Restricted Fullrank Single-symbol Decodable Design for Four Transmit Antennas," IEEE Signal Processing Letter, in press.

[9] J. Wu and S. D. Blostein, "High-rate codes over space, time, and frequency," IEEE Globalcommunications Conference (GLOBECOM 2005), Mohsen Guizani, USA, vol. 6, December 2005.

[10] H. Lee, J. Cho, J. Kim and I. Lee, "An efficient decoding algorithm for STBC with multi-dimensional rotated constellations," IEEE International Conference on Communications (ICC 2006), Istanbul, Turkey, June 2006.

[11] R. Grover, W. Su and D.A. Pados, "PEP-bound rotation angle optimization of 8-transmit-antenna quasi-orthogonal space-time block codes," IEEE Acoustics, Speech and Signal Processing (ICASSP 2007), Honolulu, Hawai'i, U.S.A, Vol. 3, April 2007. 